\title{
THE RÔLE OF THE CONCEPT OF INFINITY IN THE WORK OF LUCRETIUS.
}

BY PROFESSOR C. J. KEYSER.

(Read before the American Mathematical Society December 27, 1917.)

No doubt there will one day be written the history of the concept of infinity. If it is to be done by an American scholar, it will probably not be done in the present generation, for the doing of it calls for a kind of composite scholarly preparationlinguistic, historical, philosophical, scientific, and especially mathematical-which our American universities have indeed the machinery but not yet, it seems, the spirit or the purpose or the atmosphere or the temper to provide.

In any adequate historical survey of the rôle of the notion of infinity in our human thinking a consideration of the De Rerum Natura of Lucretius will have to be accorded the position of an important chapter. Most of the many great merits of the work have been long, if not generally nor even widely, recognized. One of its recognized merits is its superb daring and the unsurpassed magnificence of its enterprise; another is its probably unmatched union of literary excellence with scientific spirit and aim; still another, which includes many, being a highly composite merit, is its confident and often acutely argued presentation, sometimes in detail and sometimes in clear and striking outline, of ideas and doctrines that came into their own only in modern science. I refer to such concepts and dogmas as natural law, the atomic constitution of matter, the conservation of mass and of energy, organic evolution, spontaneous or chance variation of life forms, struggle for existence, survival of the fit, and sensation as the ultimate basis of knowledge and the ultimate test of reality, not to mention other equally brilliant anticipations of modern scientific thought.

In attempts to appraise the work of Lucretius his employment of the notion of infinity is commonly indicated, but only more or less incidentally. For example, in Masson's large volume, Lucretius, Epicurean and Poet, the term infinite has only a subordinate place in the index of important terms; in 
Munro's very extensive Notes the term receives but scant attention; and it receives even less in the Notes found in Cyril Bailey's recent and deservedly much praised English translation of the poem. What is missed in such appreciations and commentaries and what I wish to signalize here is the fact that the concept of infinity-of infinite multitude and infinite magnitude - is not merely one among the many ideas, but is indeed the dominant idea, in the work of Lucretius. A critical examination of the work cannot fail to discover that in the author's judgment the concept in question was at once the most powerful of his instruments and the one most obviously indispensable to the success of his great undertaking. That undertaking was a pretty large contract, being nothing less than the endeavor to show forth a method by which it would be possible to explain, or to account for, all phenomena (whether mental or not) without having to resort to the hypothesis of divine intervention.

This is not the place to give a detailed account of the Lucretian principles and procedure. For the purpose of this note it is sufficient to point out that among the fundamental propositions there are three major propositions and that these owe their efficacy and their dominance of the entire discourse to the fact of their postulating the existence of infinite multitude and infinite magnitude. These propositions are that the universe of space is a region or room of infinite extent; that time is an infinite duration composed of an infinite past and an infinite future; and that the matter in the universe is composed of an infinite multitude of absolutely solid (non-porous) and non-decomposable atoms or "seeds of things" always moving hither and thither in an infinite variety of ways and ever so distributed throughout the whole of space that of all spheres none but such as are microscopically minute could at any given instant fail to enclose one or more of the "seeds." Without these postulated infinites explanation of the phenomena of the world was, in the belief of Lucretius, impossible; with them, supplemented by certain other postulates, such explanation was possible. Though the mentioned postulates were not in themselves sufficient, they were regarded as conspicuously necessary. In the view of Lucretius cosmic history was an eternal drama enacted by an infinitude of unoriginated and indestructible elements operating upon an infinite stage. The drama was not to be understood except by help of the 
concept of infinity; and so the De Rerum Natura may be not unjustly said to be a kind of poetic celebration of what the author deemed to be the scientific efficacy of that concept.

What did Lucretius mean by infinity? What did he mean by an infinite multitude and by an infinite magnitude? No formal definition of any of these terms is to be found in his work. But it is perfectly clear that he conceived an infinite multitude of elements to be a multitude which could not be exhausted by removing from it one element at a time but which could be thought as arranged in an endless succession of elements. In other words, an infinite multitude signified what we now describe as a denumerably infinite multitude. There is no hint at any conception of any higher order of infinity.

It is noteworthy that, with the possible exception of time, the fundamental infinites of Lucretius were not mere variables capable of increase beyond any prescribed finite amount; on the contrary, they were, like the infinites of Cantor, constant or static affairs; but, unlike the Cantor infinites, those of Lucretius were composed of actual, or concrete, things and not abstract ones like points, for example, or pure numbers; thus the Lucretian infinitude of atoms were material particles and they all existed at once.

Was Lucretius aware of the fact that an infinite multitude, as conceived by him, contained parts or submultitudes equivalent, as we now say, to the whole? He was not only aware of it but he repeatedly employed this characteristic property of infinity effectively and correctly. This rather astonishing fact is sufficiently interesting to justify citation of one or two of the numerous passages supporting its assertion. If we bear in mind that one of the Lucretian infinites was the succession of time units (days or generations or other stretches) beginning at any given instant and together composing what is called the future, the following famous passage makes it perfectly clear that, according to its author, the removal of any finite multitude of elements from an infinite multitude of them leaves a remainder, a part, exactly equal or equivalent to the whole:

"Nor by prolonging life do we take one tittle from the time past in death nor can we fret anything away, whereby we may haply be a less long time in the condition of the dead. Therefore you may complete as many generations as you please; 
none the less however will that everlasting death await you; and for no less long a time will he be no more in being, who beginning with today has ended his life, than the man who has died many months and years ago." *

Lucretius, as already said, postulated the existence of an infinity of atoms. These were not all of them identical in shape or in size but in these respects presented a finite number of varieties. Each variety, it was further assumed, constituted an infinite multitude. $\dagger$ These infinites were not distinguished as such; in respect of multiplicity, though not in respect of their physical functions, they were equivalent to one another and to the whole multitude constituted by their elements.

It is sufficiently evident that the poet's conception of infinite multitude was identical with that now employed by mathematicians. Careful scrutiny of the poem will discover that the same may be said of the author's conception of infinite magnitude. Formal definition of the notion is not present. We are told, however, that in respect of size, all the atoms are between a finite upper bound and a finite lower bound; we are told that the sum of any finite number of atoms is finite; we are told that the sum of all atoms of a given form is infinite and that, therefore, the number of them must be infinite. It is thus evident that for Lucretius an infinite magnitude is a magnitude greater than the sum of any finite number of finite quantities none of which surpasses, in respect of parvitude, a finite size.

Formation or possession of ideas is one thing; logically correct handling of them in argumentation is quite another. In his use of the ideas in question Lucretius was sometimes right and sometimes wrong. Of right use some examples have already been given and it would be easy to cite others. As an instance of erroneous use the following passage (as correctly translated by Munro, page 15) may be cited on account of its great interest:

"Again unless there shall be a least, the very smallest bodies will consist of infinite parts, inasmuch as half of a half will always have a half and nothing will set bounds to the division. Therefore between the sum of things and the least of things

* Munro's Lucretius, 4th ed., p. 83.

$\dagger$ Cf. Munro, pp. 39, 40,41, or Bailey's Lucretius On the Nature of Things, pp. $76,83,84$. 
what difference will there be? There will be no distinction at all; for how absolutely infinite soever the whole sum is, yet the things which are smallest will equally consist of infinite parts."

The significance of the passage and the nature of the error contained in it will be clearer if we note that it is a portion of the argument by which the author seeks to establish his contention that among the parts composing an atom (which is by assumption the smallest material particle capable of existing spatially separate from all other particles) there is a least part, by which is meant a part so small that none of the parts is smaller. Paraphrased in modern terms this portion of the argument would run about as follows: if among the parts composing an atom and being such that no two of them have points in common save points of a common surface there be no least part, then the atom consists of an infinite number of parts; the number of atoms in the universe is infinite; these two infinite multitudes of finite portions of matter are equivalent; the sum of the elements of the latter multitude is an infinite magnitude; such, too, is the sum of the elements of the former multitude; but this sum is the atom itself; hence, unless there be a least part among the parts of an atom, an atom is an infinite magnitude and as such is no less than the sum of all matter. The error is not due to a wrong conception of infinity, whether of multitude or of magnitude, but is obviously due solely to the tacit assumption of the false proposition that the sum of the elements of any infinite multitude of finite elements is infinite. An obvious moral is that a little knowledge of the convergence of series would greatly improve the philosophy of poets and the science of philosophers.

It is astonishing that the mentioned fallacy occurs in immediate conscious connection with a line seeming to refute it: "the half of the half will always have a half." What is the explanation? It is not to be found in any supposition of stupidity or of momentary nodding. It is to be found in the author's purpose and point of view. $\mathrm{He}$ was exclusively concerned with natural phenomena, with what he deemed to be existing entities. Regarding the series, $\frac{1}{2}, \frac{1}{4}, \frac{1}{8}, \cdots$, he would have said in effect: "Composed of man-made symbols like words, it is not nor ever can be endless; to speak of the sum of a non-existing endless series is meaningless; moreover, even if we supposed the series to be endless, to be summable, 
and to have 1 for its sum, this 1 would be neither finite nor infinite, for it would not be a magnitude, inasmuch as the summands are themselves not magnitudes but are merely empty abstract symbols; if 1 be said to be a magnitude, in the sense of representing a magnitude, then, if the magnitude 1 be composed of two equal magnitudes, $\frac{1}{2}$ will be a magnitude in the same sense, and so on for the other symbols; if all the symbols be magnitudes in the indicated sense, the summation of the abstract series will be the summation of an endless series of magnitudes; otherwise not; and now what I have contended in my book is that, if the magnitude 1 be finite, not more than a relatively few of the symbols of the series can be magnitudes, and this contention, denying the infinite divisibility of finite magnitude, is based on grounds other than that advanced in the above-quoted passage from my argument." This is not the place to recount, much less to estimate, those "other" grounds. It must, however, be said, in passing, that one of them is in point of kind almost perfectly indicated by the following words of Clerk Maxwell:* "What we assert is that after we have divided a body into a certain finite number of constituent parts called molecules, then any further division of these molecules will deprive them of the properties which give rise to the phenomena observed in the substance."

The classic form of the false thesis tacitly invoked by Lucretius in the above-quoted passage to fortify his "other" grounds for denying the infinite divisibility of a finite magnitude is exceedingly vague: all infinities are equal. It has had an age-long and world-wide vogue. Thus Kanadi, an old Hindoo author, employs the thesis to prove that, if every body be infinitely divisible, there can be "no difference of magnitude between a mustard seed and a mountain." $\dagger$ In this connection mathematicians, especially those who may be interested in the history of the notion of infinity, will be glad to have their attention called to a little-known letter $\ddagger$ of Newton dealing with the matter. The letter, which is addressed to Richard Bentley, is very interesting on several accounts: it points out the vagueness and the falsity of the above-mentioned thesis, which Dr. Bentley had assumed to be true; it repeatedly employs the term infinite in a sense not less

\footnotetext{
* Theory of Heat, p. 285.

$\dagger$ See Daubeny's Introduction to the Atomic Theory, p. 5.

$\ddagger$ See The Works of Richard Bentley, vol. III, p. 207.
} 
vague and indeterminate; it virtually asserts that, if two infinite magnitudes be equal, the addition of any finite magnitude to either of them will destroy the equality.

In closing this note I desire to guard against the danger of leaving a false impression. The mere correctness of the Lucretian concept of infinity by no means accounts for the immense rôle of the concept in the author's work. The secret lies in the fact that the concept so powerfully stimulated the imagination of a great thinker and poet as to cause him to express and to preserve in immortal form a body of ideas which he had acquired from the then still extant works of Epicurus and which after the long lapse of centuries are found to be among the most fruitful scientific ideas of our time.

Columbia UnIVersity, December, 1917.

\section{ON THE INVARIANT NET OF CUBICS IN THE STEINERIAN TRANSFORMATION.}

BY PROFESSOR ARNOLD EMCH.

(Read before the American Mathematical Society September 4, 1917.)

1. By Steinerian transformation* we understand an involutorial quadratic Cremona transformation, defined as the one-to-one correspondence between the points of a plane (with the exception of the points of a certain trilateral) and the points of concurrence of their polars with respect to the conics of a pencil. If we use the base points $A_{1}, A_{2}, A_{3}, E$ of the pencil as the vertices and the unit point of a system of projective coordinates, the Steinerian transformation may easily be established in the form

$$
\begin{aligned}
& \rho x_{1}^{\prime}=x_{1}\left(x_{2}+x_{3}-x_{1}\right), \\
& \rho x_{2}^{\prime}=x_{2}\left(x_{3}+x_{1}-x_{2}\right), \\
& \rho x_{3}{ }^{\prime}=x_{3}\left(x_{1}+x_{2}-x_{3}\right) .
\end{aligned}
$$

The base points $A_{1}(1,0,0) ; A_{2}(0,1,0) ; A_{3}(0,0,1) ; E(1,1$,

* See Annals of Mathematics, vol. 14 (1912), pp. 57-71. 